\title{
Programa de intervenção e atenção precoce com bebês na Educação Infantil
}

\section{Early intervention program in Nursery School with babies}

\author{
Carolina Santos Soejima ${ }^{1}$ \\ Maria Augusta Bolsanello ${ }^{2}$
}

\begin{abstract}
RESUMO
A pesquisa ${ }^{3}$ verificou se um programa de intervenção precoce tem efeitos positivos sobre o desenvolvimento (motor e mental) de crianças entre um e três anos de idade que permaneciam em tempo integral na creche pública e apresentavam defasagem no desenvolvimento. Inicialmente, foram avaliadas 63 crianças por meio das Escalas Bayley II, sendo que 37 delas apresentaram defasagem no desenvolvimento em ambas as escalas, mental e motora. Destas 37 crianças, 20 foram sorteadas para participar de um programa de intervenção precoce. Após a realização desta intervenção, foram sorteadas, para a segunda aplicação das Escalas Bayley II, 15 crianças com defasagem e participantes da intervenção, 15 crianças com defasagem e não participantes da intervenção e 15 crianças sem defasagem. Os resultados evidenciaram visível progressão no desenvolvimento das crianças que apresentaram defasagem e participaram da intervenção precoce. Concluiu-se a viabilidade do emprego de uma intervenção de caráter preventivo nas creches públicas, na perspectiva da atenção precoce. Dessa forma, defasagens do desenvolvimento infantil podem ser prevenidas, identificadas, trabalhadas e superadas, evitando futuras deficiências ou transtornos no desenvolvimento infantil.
\end{abstract}

Palavras-chave: desenvolvimento infantil; educação infantil; atenção precoce; intervenção precoce.

${ }^{1}$ Doutora em Educação pela Universidade Federal do Paraná. Professora da Faculdade Metropolitana de Blumenau, Fameblu/Uniasselvi, de Santa Catarina, Brasil. E-mail: carolinasoejima@hotmail.com.

${ }^{2}$ Doutora em Psicologia Escolar e do Desenvolvimento Humano pela Universidade de São Paulo. Professora Associada da Universidade Federal do Paraná, Brasil. E-mail: mabolsanello@ufpr.br.

${ }^{3}$ Pesquisa com apoio do CNPq (Conselho Nacional de Desenvolvimento Científico e Tecnológico), Brasil. 


\begin{abstract}
The research examined whether a program of early intervention has positive effects on the development (motor and mental) in children with ages from one to three who stay full-time in a public nursery school and present developmental delay. Initially 63 children were evaluated using the Bayley Scales II, being that 37 of them showed delay in both scales, mental and motor. Of these 37 children, 20 were randomly selected to participate in an early intervention program. After this intervention, three groups were formed for the second application of the Bayley Scales II, being that the groups were compounded of 15 children with developmental delay who had participated in the mentioned early intervention, 15 children who did not participate in the intervention but had developmental delay, and 15 children who did not have developmental delay. The results showed visible progress in the development of children with developmental delay who had participated in the early intervention. The study concluded that a program of early intervention in public nursery schools is viable to prevent future handicaps in children because the early intervention can detect and identify possible mental or motor underdevelopment and provide means to overcome them.
\end{abstract}

Keywords: child development; early childhood education; nursery; early intervention.

Nas últimas décadas, sobretudo nos Estados Unidos e países europeus, os programas de estimulação precoce sofreram uma transformação notável. Dentro do novo enfoque, a estimulação precoce toma uma nova denominação - atenção precoce (do espanhol atención temprana) -, consistindo em um conjunto de intervenções dirigidas à população infantil de zero a seis anos de idade, à família e ao contexto, tendo por objetivo responder às necessidades transitórias ou permanentes que apresentam crianças de risco ou com transtornos em seu desenvolvimento (GAT, 2000). Desta maneira, a atenção precoce passa a ser considerada como um conjunto de serviços, apoios e recursos que são necessários para responder tanto às necessidades de cada criança quanto às necessidades de suas famílias, sempre visando à promoção do desenvolvimento infantil (DUNST; BRUDER, 2002).

A qualidade do ambiente familiar e educacional que se pode oferecer à criança, desde seu nascimento, tem efeito posterior nas distintas etapas do desenvolvimento infantil (SHONKOFF; MEISELS, 2000). Um cuidado inadequado pode levar a uma situação de risco ou atraso evolutivo (GURALNICK, 1997). Pensa-se, então, em prevenção em todos os níveis (OMS, 1980): da prevenção primária, antes de qualquer indício de deficiência, risco ou atraso; da prevenção secundária, quando há algum indício de deficiência, risco ou 
atraso; e da prevenção terciária, que trata de minimizar os efeitos e reduzir ao máximo o transtorno ou deficiência constatada. A prevenção, de qualquer nível, será efetiva quando se fundamentar em teorias sólidas, incluir a criança, sua família e a escola e ser conduzida por pessoas capacitadas, capazes de seguir sistematicamente programas e procedimentos de intervenção (BRITO DE LA NUEZ et al., 2006). Além disso, a prevenção deve ser adequada à cultura e à idade das crianças.

O principal objetivo da Atenção Precoce (AP), a partir da vertente preventiva, é fazer com que as crianças que apresentam transtornos em seu desenvolvimento ou possuem o risco de vir a apresentá-los recebam as intervenções necessárias para promover e potencializar o seu desenvolvimento, possibilitando-lhes sua integração no ambiente familiar, escolar e social, assim como sua autonomia pessoal (GAT, 2000). Desta feita, os principais âmbitos de atuação da Atenção Precoce são os serviços de saúde, sociais e educativos.

A escola infantil está estreitamente vinculada à atenção precoce, pois ambas possuem o mesmo objetivo, que é fomentar o desenvolvimento global da criança. Estar atenta aos fatores emocionais, físicos, sociais e cognitivos significa prevenir eventuais atrasos do desenvolvimento e compensar determinadas carências que podem advir do ambiente familiar (MULAS, 2007). Em situações de risco, a presença de um transtorno e/ou deficiência é detectada por educadores ou professores de creche. Não se pode esquecer que fatores pré, peri e pós-natais possuem alta incidência junto às populações de baixa renda, envolvendo crianças que estão nas creches públicas, o que justifica procedimento de acompanhamento do desenvolvimento na primeira infância (BRITO, 2004; CARAVACA, 2006; ROSSETTI-FERREIRA; RAMON; SILVA, 2002).

Por conseguinte, Brito de La Nuez et al. (2006) também consideram pertinente estender programas de avaliação e intervenção precoce às crianças expostas aos riscos socioambientais (situações de maltrato, abuso e negligência), com intuito de estimular o desenvolvimento da criança, suas competências e, deste modo, elevar sua qualidade de vida e de suas famílias.

Observa-se que, em vista de suas particularidades, a educação infantil tem um forte componente de prevenção, mas também deve primar por sua qualidade. Neste sentido, Lindsey (1998) aponta que escolas infantis de baixa qualidade podem propiciar o surgimento de defasagens no desenvolvimento. Estudos têm salientado estas defasagens em crianças que permanecem em tempo integral na creche (DUNCAN, 2003, VOTRUBA-DRZAL; COLEY; CHASE-LANSDALE, 2004). Desta feita, é de fundamental importância considerar os efeitos das condições precárias do ambiente da creche (espaços inadequados, falta de apoio pedagógico, muitas crianças, professores estressados, entre outras) sobre a experiência da criança pequena. A creche precisa ser um ambiente educativo 
valorizado, para que o acesso aos bens culturais seja facilitado e oferecido à criança, estimulando o seu desenvolvimento (MOREIRA; LORDELO, 2002; PACHECO; DUPRET, 2004; VOLPATO; MELLO, 2005; SOUZA, 2008). Nesse sentido, pais e educadores são essenciais em otimizar o desenvolvimento infantil, aproveitando os momentos cotidianos e naturais para fomentar a qualidade da estimulação e interação entre eles. É preciso auxiliar pais e educadores a potencializar a reciprocidade e a atenção conjunta, estar atentos aos sinais das crianças, serem contingentes e mostrar sincronia em suas respostas, além de participar e promover jogos e brincadeiras adequados às capacidades da criança (CARAVACA, 2006; KRAUSS, 2000; PÉREZ-LÓPEZ, 2004; PÉREZ-LÓPEZ et al., 2006; CANDEL, 2004; RAPOPORT, 2003).

Os estímulos que rodeiam a criança devem ser adequados às suas capacidades e deve-se evitar, portanto, a persistência nos estímulos já suficientemente experimentados e processados, que poderiam resultar repetitivos e pouco desafiadores (EZPELETA, 2005).

A atenção precoce, por sua vez, não pode ser vista, exclusivamente, sob a vertente da reabilitação, da intervenção psicossocial ou da educação. Todavia, deve fazer parte de um processo integral que tem como fim último o desenvolvimento harmônico de crianças integradas aos contextos em que vivem.

Com estas considerações, o presente estudo buscou verificar se um programa de intervenção precoce tem efeitos positivos sobre o desenvolvimento (motor e mental) de crianças entre zero e três anos de idade que permaneciam tempo integral em creche pública.

\section{Método}

\section{Contexto}

A pesquisa desenvolveu-se em um Centro de Educação Infantil público, de uma cidade de Santa Catarina, cuja clientela eram crianças de famílias trabalhadoras de baixa renda. Enfocou três turmas de crianças entre zero e três anos de idade que permaneciam em tempo integral no referido Centro (aqui denominado de creche), das 7 às 19 horas. A instituição e os responsáveis pelas crianças assinaram o Termo de Consentimento Livre e Esclarecido para participação na pesquisa, aprovado pelo Comitê de Ética em Pesquisa do Setor de Ciências da Saúde da Universidade Federal do Paraná. 


\section{Participantes}

\section{Participantes e instrumentos da primeira etapa da pesquisa}

Participaram da primeira parte da pesquisa 63 crianças (aqui também denominadas de bebês), distribuídas em três turmas. Eram 20 crianças entre zero e 11 meses (Berçário), 20 crianças entre 12 e 23 meses (Maternal I) e 23 crianças entre 24 e 36 meses (Maternal II). As crianças foram avaliadas por meio das Escalas Mental e Motora de Desenvolvimento Infantil Bayley II (BAYLEY, 1993), também conhecidas como Escalas Bayley II.

As Escalas Bayley II (BAYLEY, 1993) consistem em um instrumento para a avaliação do desenvolvimento de crianças entre um e 42 meses de idade e abrangem duas escalas, mental e motora, além de um registro do comportamento infantil. A Escala Mental destina-se à avaliação de acuidades sensório-perceptuais, discriminações, memória, aprendizagem, solução de problema, início da comunicação verbal, generalizações e classificações. A Escala Motora fornece uma medida do grau de controle do corpo, coordenação dos grandes músculos e habilidades manipulatórias mais finas das mãos e dos dedos. As Escalas possibilitam identificar crianças com atraso evolutivo e proporcionar informação para planejar estratégias de intervenção. É de fácil aplicação, consiste em pequenas provas em forma de brincadeiras, utilizando materiais simples que despertam a atenção e a curiosidade das crianças.

As avaliações foram realizadas individualmente com cada criança, na presença do cuidador principal (mãe, pai ou responsável), em sala adequada do Centro de Educação Infantil. A presença do cuidador principal visa dar suporte e segurança à criança e ainda auxiliar na aplicação de algumas provas (BAYLEY, 1993).

No presente artigo, considerou-se defasagem no desenvolvimento uma idade equivalente de desenvolvimento (IED) dois meses aquém da idade cronológica da criança, conforme preconiza o manual das Escalas Bayley de Desenvolvimento Infantil II (BAYLEY, 1993). Complementando, Brito de la Nuez et al. (2006) definiram defasagem como carências ou atrasos no desenvolvimento.

As Escalas Bayley II não foram adaptadas para a população brasileira. Diante disso, os resultados das avaliações das crianças foram comparados entre si, nos dois momentos de aplicação. Crianças prematuras tiveram a sua idade corrigida por meio do cálculo de ajuste indicado pelo Manual das Escalas Bayley II.

Das 63 crianças submetidas à primeira aplicação das Escalas Bayley II, 37 apresentaram defasagem no desenvolvimento em ambas as escalas do instrumento. 


\section{Participantes da segunda etapa da pesquisa: Intervenção em Estimulação Precoce}

Das nove crianças do Berçário que apresentaram defasagem no desenvolvimento, cinco foram sorteadas para participar de um programa de intervenção precoce e quatro não foram participantes; das 15 crianças do Maternal I que apresentaram defasagem, oito foram sorteadas para participar da intervenção precoce e sete não foram participantes; e, finalmente, das 13 crianças do Maternal II que apresentaram defasagem no desenvolvimento, sete foram sorteadas para participar da intervenção precoce e seis não foram participantes. Por conseguinte, das 37 crianças que apresentaram defasagem no desenvolvimento, 20 foram sorteadas para participar do programa de intervenção precoce.

O programa de intervenção precoce foi elaborado individualmente para cada bebê, de acordo com a literatura sobre desenvolvimento infantil e intervenção precoce (BAYLEY, 1993; BUGIÉ; LORENTE, 2003; CARAVACA, 2006; GAT, 2000; MILLÁ; MULAS, 2005; PÉREZ-LÓPEZ, 2004; PÉREZ-LÓPEZ et al., 2006; ATEMP, 2002). Foram utilizadas as orientações empregadas em estimulação e intervenção precoce, valorizando a idade, as necessidades e o desenvolvimento da criança. A intervenção compreendeu atividades lúdicas com objetivos de desenvolver áreas específicas. Para a sua efetivação foram utilizados brinquedos diversos, desenvolvidos materiais variados e utilizados recursos disponíveis na própria creche.

A intervenção precoce foi desenvolvida por uma das pesquisadoras do presente artigo, durante um mês, de segunda a sexta-feira, na própria creche dos bebês, em sala adequada, no horário das 8 às 19 horas. A intervenção foi individual, teve duração aproximada de 20 minutos e todas as 20 crianças foram estimuladas.

A duração de apenas um mês da intervenção foi um critério da pesquisa, tendo em vista que as crianças da faixa etária pesquisada desenvolvem-se rapidamente e, se a duração da intervenção fosse superior ao período decidido, poderia ficar difícil analisar se os avanços no desenvolvimento do bebê ocorreram devido à intervenção ou à dinâmica própria e contextual do desenvolvimento infantil. Logo, a intervenção de curta duração, apenas um mês, provavelmente permitiu uma análise mais apurada da intervenção precoce. 


\section{Participantes e instrumento da terceira etapa da pesquisa}

Na terceira etapa da pesquisa, ou seja, na segunda aplicação das Escalas Bayley II, por meio de sorteio, foram avaliadas 15 crianças sem defasagem (cinco do Berçário, cinco do Maternal I e cinco do Maternal II); 15 crianças com defasagem e não participantes da intervenção precoce (cinco do Berçário, cinco do Maternal I e cinco do Maternal II); e 15 crianças com defasagem e participantes da intervenção precoce (cinco do Berçário, cinco do Maternal I e cinco do Maternal II). Logo, 45 crianças foram submetidas à segunda aplicação das Escalas Bayley II.

\section{Análise dos dados}

Os dados foram analisados quantitativamente, por meio da idade equivalente em meses que cada criança obteve nas Escalas Bayley II, nos dois momentos de aplicação. A análise quantitativa consistiu na comparação das idades da criança em relação consigo mesma, ou seja, foram comparadas as idades correspondentes às escalas mental e motora na primeira aplicação das Escalas Bayley II com as idades correspondentes na segunda aplicação. Além disso, utilizou-se uma medida capaz de verificar a promoção do desenvolvimento infantil, considerando o desenvolvimento individual de cada criança avaliada. Para tanto, fez-se necessária a utilização de uma variável, denominada Progresso (PÉREZ-LÓPEZ et al., 2006). Esta foi obtida a partir da diferença, para cada bebê, para cada escala e nos dois momentos de aplicação, entre a Idade Equivalente de Desenvolvimento (IED), obtida por meio das Escalas Bayley II e a Idade Cronológica (IC), dividida pela Idade Cronológica (IC) e multiplicada por 100 (BRITO DE LA NUEZ et al., 2006), como se indica na seguinte fórmula:

$$
\text { Progresso }=\frac{\text { IED }- \text { IC }}{\mathrm{IC}} \times 100
$$

Posteriormente, os dados indicativos do progresso foram submetidos às provas $t$ de Student de diferenças de médias para amostras relacionadas, entre os dois momentos de avaliação e para as duas escalas Bayley II, mental e motora.

A interpretação da equação Progresso supõe que um valor positivo indica que a idade de desenvolvimento da criança é superior à sua idade cronológica, enquanto um valor negativo indica que a idade de desenvolvimento é inferior à cronológica. Um valor próximo a zero sugere que a idade de desenvolvimento da criança se aproxima à da sua idade cronológica (BRITO DE LA NUEZ et al., 2006). 


\section{Resultados e discussão}

A análise dos dados indicou a existência de diferenças, algumas significativas, nas comparações realizadas, como se evidencia nas figuras abaixo.
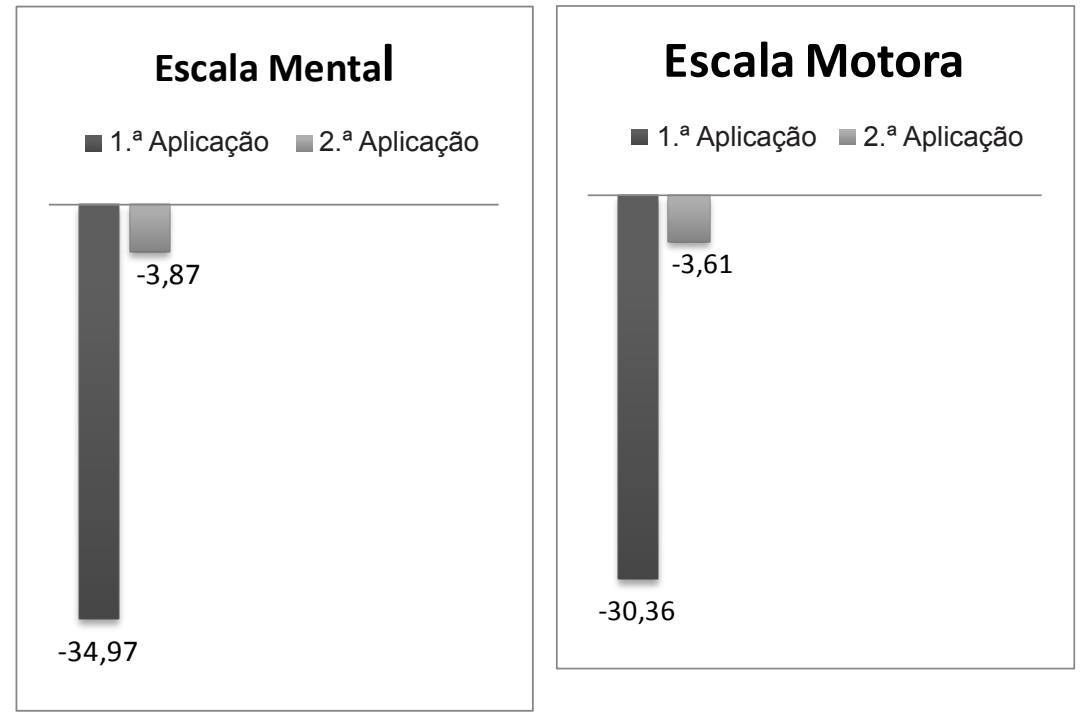

FIGURAS 1 E 2 - Comparação das médias das escalas mental e motora nas duas aplicações das Escalas Bayley II em crianças com defasagem que participaram da intervenção precoce.

FONTE: As autoras (2011)

A Figura 1 destacou as médias da escala mental nos dois momentos de avaliação das 15 crianças que apresentaram defasagem na primeira aplicação das Escalas Bayley II, participaram da intervenção precoce e foram submetidas à segunda aplicação das Bayley II $(\mathrm{p}=0,0000000969)$. A progressão das crianças da primeira para a segunda aplicação, após a intervenção precoce, foi visível e estatisticamente significativa.

A Figura 2 salientou as médias da escala motora nos dois momentos de avaliação das 15 crianças que apresentaram defasagem na primeira aplicação das Escalas Bayley II, participaram da intervenção precoce e foram submetidas à segunda aplicação das Bayley II $(\mathrm{p}=0,00000045)$. Similarmente à escala mental, percebeu-se significativa progressão entre as aplicações, após a intervenção. 
A evolução notória na Figura 1 e na Figura 2 levou a inferir que a intervenção precoce influencia o desenvolvimento infantil, respondendo às necessidades transitórias ou permanentes que apresentam as crianças com defasagem (GAT, 2000).
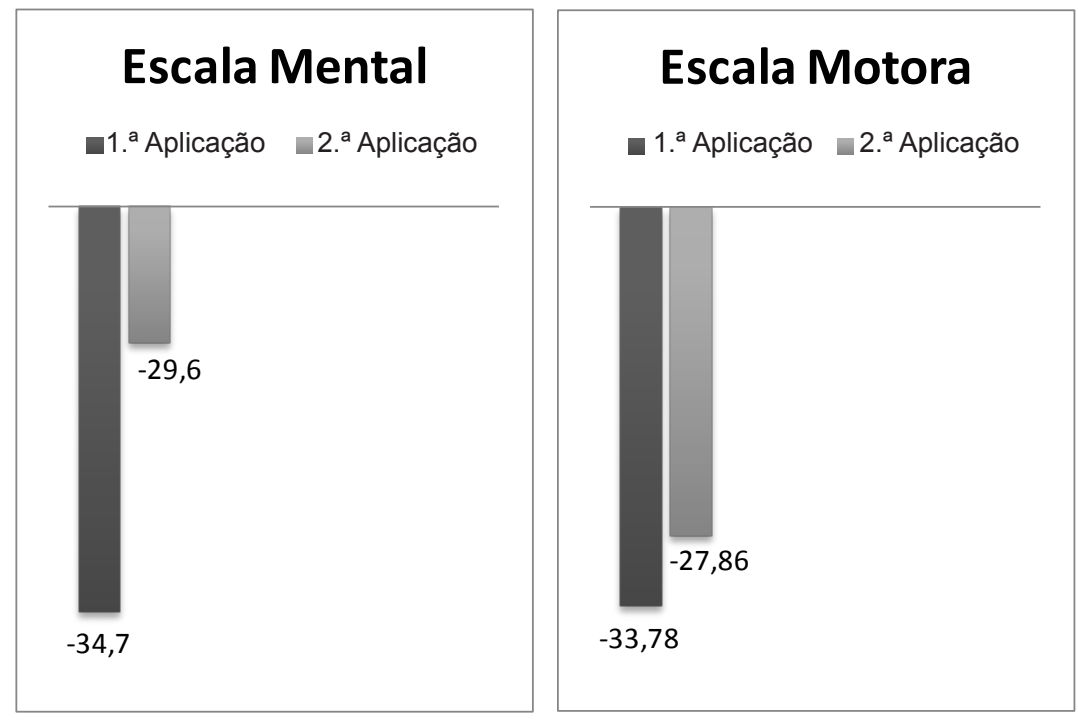

FIGURAS 3 E 4 - Comparação das médias das escalas mental e motora nas duas aplicações das Escalas Bayley II em crianças com defasagem e que não participaram da intervenção precoce.

FONTE: As autoras (2011)

A Figura 3 exibiu as médias da escala mental nos dois momentos de avaliação das 15 crianças que apresentaram defasagem na primeira aplicação das Escalas Bayley II, mas que não participaram do programa de intervenção precoce $(p=0,0143)$. Observou-se progressão na escala mental; entretanto, essa é menor do que a evidenciada nas crianças que participaram da intervenção precoce.

A Figura 4 destacou as médias da escala motora nos dois momentos de aplicação das Escalas Bayley II das 15 crianças que apresentaram defasagem, mas que não participaram da intervenção em estimulação precoce $(p=0,0104)$. Observou-se certa progressão, porém bem menos acentuada que a evidenciada no grupo de crianças que participaram do programa de intervenção precoce.

A pequena progressão entre a primeira e a segunda aplicação das Escalas Bayley II em ambas as escalas, mental e motora, muito provavelmente pode 
ser resultante da própria dinâmica do desenvolvimento infantil (BRITO, 2004; BRITO DE LA NUEZ et al., 2006; EICKMANN; LIRA; LIMA, 2002). É possível também que tenha sido influenciada por a criança já conhecer e estar familiarizada com o instrumento e a examinadora (BAYLEY, 1993) e, finalmente, por ter recebido maior estimulação da educadora ou dos pais no período que compreendeu a primeira e a segunda avaliação do desenvolvimento (ANJOS et. al., 2004; BARROS; FRAGOSO; OLIVEIRA, 2003; BÓGUS; NOGUEIRA-MARTINS; MORAES; 2007; JUAN-VERA; PÉREZ-LÓPEZ; CASELLAS, 2006; LORDELO; CHALBUD; GUIRRA; CARVALHO, 2007).
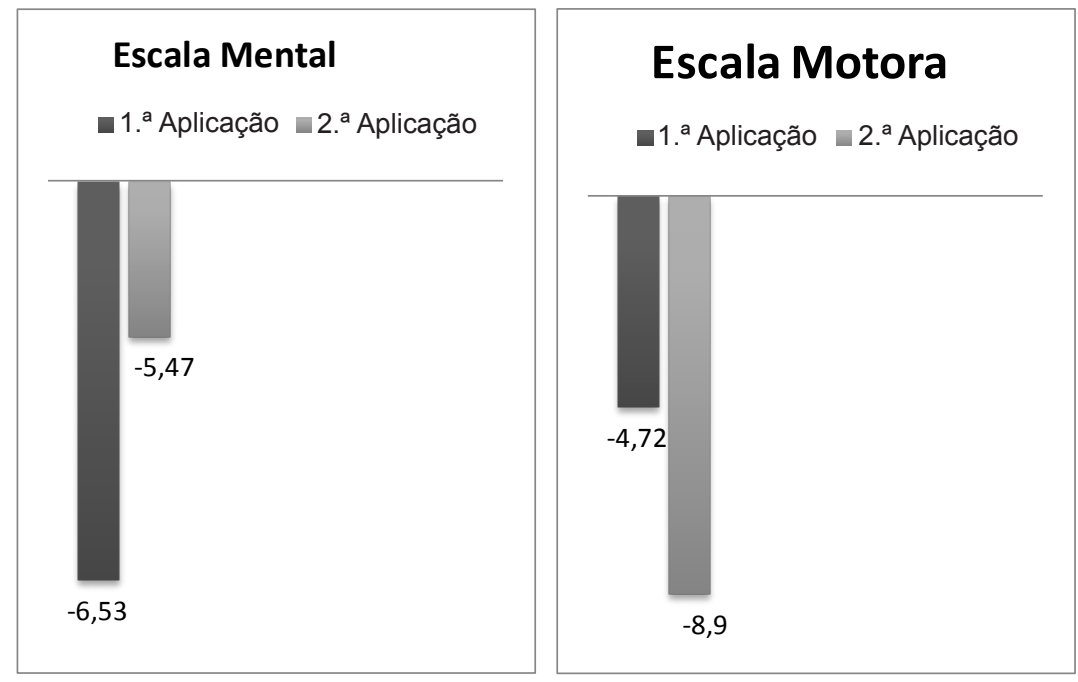

FIGURAS 5 E 6 - Comparação das médias das escalas mental e motora nas duas aplicações das Escalas Bayley II nas crianças sem defasagem.

FONTE: As autoras (2011)

A Figura 5 mostrou as médias da escala mental nos dois momentos de aplicação das Escalas Bayley II das 15 crianças que não apresentaram defasagem $\mathrm{e}$, portanto, não participaram do programa de intervenção precoce $(\mathrm{p}=0,23)$. Observou-se certa progressão, porém essa diferença não foi estatisticamente significativa, como a diferença encontrada nas médias da escala mental das crianças que participaram da intervenção precoce - que é significativa estatisticamente $(\mathrm{p}=0,0000000969)$.

A Figura 6 comparou as médias da escala motora nos dois momentos de aplicação das Escalas Bayley II das 15 crianças que não apresentaram defasagem 
e, portanto, não participaram da intervenção precoce $(p=0,000869)$. Diferentemente da escala mental, não se observou evolução entre os dois momentos de avaliação. As crianças sem defasagem apresentaram média inferior na segunda aplicação das Escalas Bayley II na escala motora.

Como foi possível observar, por meio das figuras apresentadas, a progressão mais evidente foi no grupo de crianças que apresentou defasagem e que participou do programa de intervenção precoce. Os outros grupos - crianças com defasagem, mas que não participaram da intervenção precoce e crianças sem defasagem - apresentaram alguma ou nenhuma progressão. Logo, a notória progressão das crianças que participaram da intervenção precoce corroborou as pesquisas que relacionam a intervenção à potencialização do desenvolvimento infantil (BRITO DE LA NUEZ et al., 2006; BUGIÉ; LORENTE, 2003; CANDEL, 2004; CARAVACA, 2006; KRAUSS, 2000; MILLÁ; MULAS, 2005; PÉREZ-LÓPEZ, 2004; PÉREZ-LÓPEZ et al., 2006; SOEJIMA, 2008; DÍAZ-HERRERO; MARTÍNEZ-FUENTES, 2009).

Os resultados confirmaram a importância da atenção precoce para o desenvolvimento infantil, sendo corroborados pelos estudos que apontaram a pertinência de ações de prevenção e intervenção que favoreçam a qualidade do ambiente e da relação educador-criança-família para o desenvolvimento infantil (ANDRADE; SANTOS; BASTOS, 2005; BARROS; FRAGOSO; OLIVEIRA, 2003; MACEDO; ANDREUCCI; MONTELLI, 2004; MEIO; LOPES; MORSCH, 2004; MILLÁ; MULAS, 2005; BOLSANELLO, 2009).

\section{Conclusões e considerações}

Os dados evidenciaram progressão significativa no desenvolvimento das crianças que apresentaram defasagem e participaram de um programa de intervenção precoce. Os outros grupos - crianças com defasagem, mas que não participaram da intervenção precoce e crianças sem defasagem - apresentaram menor ou pouca progressão no desenvolvimento na segunda aplicação das Escalas Bayley II.

De acordo com a literatura e com os dados analisados na presente pesquisa, postula-se a viável alternativa de uma atenção precoce nas creches públicas. Dessa forma, defasagens no desenvolvimento infantil poderiam ser prevenidas, trabalhadas e superadas, evitando futuras deficiências ou transtornos do desenvolvimento. 
Ressalta-se também a necessidade de se fomentar contextos (escola, família e comunidade) sensíveis às necessidades e capacidades da criança em cada momento de seu desenvolvimento.

A presente pesquisa constitui uma das poucas disponíveis na realidade brasileira sobre a atenção precoce na creche pública. Portanto, o aprofundamento em novos estudos é necessário, considerando que a pesquisa compreendeu uma amostra pequena e, consequentemente, não generalizável. Novas pesquisas devem deter-se ao tema, visando encontrar sugestões e respostas capazes de orientar as políticas públicas, as ações institucionais e o trabalho dos profissionais envolvidos na educação infantil, sobretudo os relacionados à faixa etária de zero a três anos de idade, para que sejam evidenciadas formas pertinentes e capazes de atender com qualidade as crianças que frequentam creches públicas, na prevenção e promoção do seu desenvolvimento.

Não se pode deixar de apontar, no presente estudo, um dado que não pode ser ignorado: o grande número de crianças que apresentaram defasagem no desenvolvimento. Embora a escala aplicada ainda não esteja validada para a população brasileira, tal fato evidencia a necessidade de se legitimar instrumentos confiáveis de avaliação do desenvolvimento infantil, tão escassos no que condiz à realidade nacional.

Em conclusão, considerar a educação infantil como um recurso importante para a prevenção e promoção do desenvolvimento significa adequar o tempo e o espaço da escola infantil a uma perspectiva psicopedagógica baseada nos postulados da atenção precoce, sem deixar de lado, ao mesmo tempo, a importância de realizar ações articuladas com outros setores da sociedade igualmente responsáveis por este espaço educativo.

\section{REFERÊNCIAS}

ANDRADE, S.; SANTOS, D.; BASTOS, A. Ambiente familiar e desenvolvimento cognitivo infantil: uma abordagem epidemiológica. Revista de Saúde Pública, v. 39, n. 4, p. 606-611, 2005.

ANJOS, A. M.; AMORIM, K. S.; VASCONCELOS, C. R. F.; ROSSETTI-FERREIRA, M. C. Interações de bebês em creche. Estudos de Psicologia, v. 9, n. 3, p. 28-45, 2004.

ATEMP. Criterios de calidad estimular: para niños de 0 a 3 años. v. 1 e 2. Murcia, España: IMAS, 2002. 
BARROS, K.; FRAGOSO, A.; OLIVEIRA, A. Influências do ambiente podem alterar a aquisição de habilidades motoras? Uma comparação entre pré-escolares de creches públicas e escolas privadas. Arquivos de Neuro-Psiquiatria, v. 61, n. 2, p. 170-175, 2003.

BAYLEY, N. Bayley scales for infant development. San Antonio: The Psychological Corporation, 1993.

BÓGUS, C.; NOGUEIRA-MARTINS, M.; MORAES, D. Cuidados oferecidos pelas creches: percepções de mães e educadoras. Revista de Nutrição, v. 20, n. 5, p. 499-514, 2007.

BOLSANELLO, M. A. Prevención desde la escuela infantil: desafios em la realidad brasileña. Revista Interuniversitaria de Formación del Profesorado, v. 65, n. 23/2, p. 73-82, 2009.

BRITO, A. G. Prevención en el âmbito de las poblaciones de risgo biológico. In: PÉREZ-LÓPEZ, J.; BRITO, A. G. (Ed.). Manual de Atención Temprana. Madrid: Pirámide, 2004.

BRITO DE LA NUEZ, A.; PÉREZ-LÓPEZ, J.; MARTÍNEZ-FUENTES, M. T.; DÍAZ-HERRERO, A. La prevención primaria em escuelas infantiles desde el servicio de prevención y promoción del desarrollo infantil y atención temprana. Revista de Atención Temprana, n. 9, p. 91-94, 2006.

BUGIÉ, C.; LORENTE, I. Diagnóstico em atención temprana. Minusval, Número Especial, p. 38-47, 2003.

CANDEL, I. Prevención desde el ámbito educativo. Patologías no evidentes. In: PÉREZ-LÓPEZ, J.; BRITO DE LA NUEZ, A. G. (Ed.). Manual de Atención Temprana. Madrid: Pirámide, 2004.

CARAVACA, J. S. La eficacia de los programas de atención temprana em niños de riesgo biológico. 303 f. Tesis (Doctorado em Psicología) - Departamento de Psicología Evolutiva, Faculdad de Psicología, Universidad de Murcia, España, 2006.

DÍAZ-HERRERO, A.; MARTÍNEZ-FUENTES, M. T. Prevención y promoción del desarrollo infantil: uma experiência em las escuelas infantis. Revista Interuniversitaria de Formación del Profesorado, v. 65, n. 23/2, p. 57-72, 2009.

DUNCAN, G. Modeling the impacts of child care quality on children's preschool cognitive development. Child Development, v. 74, p. 1454-1475, 2003.

DUNST, C.; BRUDER, M. B. Valued outcomes of service coordination, early intervention and natural environments. Exceptional Children, v. 68, n. 3, p. 361-375, 2002.

EICKMANN, S.; LIRA, P.; LIMA, M. Desenvolvimento mental e motor aos 24 meses de crianças nascidas a termo com baixo peso. Arquivos de Neuro-Psiquiatria, v. 60, n. 3, p. 748-754, 2002.

EZPELETA, L. Factores de riesgo em psicopatología del desarrollo. Barcelona: Masson, 2005. 
GAT. Grupo de Atención Temprana. Libro blanco de la atención temprana. Documento 55/2000. Madrid: Real Patronato de Prevención y de Atención a Personas com Minusvalía. Ministerio de Trabajo y Asuntos Sociales, 2000.

GURALNICK, M. J. The effectiveness of early intervention. Baltimore: Paul H. Brookes Publishing, 1997.

JUAN-VERA, M.; PÉREZ-LÓPEZ, J. CASELLAS, C. E. La formación de padres como herramienta de prevención primaria em atención temprana. Revista de Atención Temprana, v. 9, p. 95-103, 2006.

KRAUSS, M. W. Family assessment within early intervention programs. In: SHONKOFF, J.; MEISELS, S. (Ed.). Handbook of early intervention. Cambridge: Cambridge University Press, 2000. p. 290-308.

LINDSEY, G. Brain research and implications for early childhood education. Childhood Education, v. 75, n. 2, p. 97-101, 1998.

LORDELO, E.; CHALBUD, A.; GUIRRA, R.; CARVALHO, C. Contexto e desenvolvimento cognitivo: Frequência à creche e evolução do desenvolvimento mental. Psicologia: Reflexão e Crítica, v. 20, n. 2, p. 324-334, 2007.

MACEDO, C.; ANDREUCCI, L.; MONTELLI, T. Alterações cognitivas em escolares de classe socioeconômica desfavorecida: resultados de intervenção psicopedagógica. Arquivos de Neuro-Psiquiatria, v. 62, n. 3, p. 852-857, 2004.

MÉIO, M.; LOPES, C.; MORSCH, D. Desenvolvimento cognitivo das crianças prematuras de muito baixo peso na idade pré-escolar. Jornal de Pediatria, v. 80, n. 6, p. 495-502, 2004.

MILLÁ, E.; MULAS, F. Atención Temprana. Valencia: Promolibro, 2005.

MOREIRA, L.; LORDELO, E. Creche em ambiente urbano pobre: ressonâncias no ecossistema desenvolvimental. InterAção, v. 6, n. 1, p. 1-13, 2002.

MULAS, J. P. Análisis de un modelo de seguimiento en atención temprana. 434 p. Tesis (Doctorado en Educación) - Facultad de Educación, Universidad Complutense de Madrid, Espanha, 2007.

OMS. Organização Mundial da Saúde. Treinando o deficiente na comunidade. Parte A. Caderno I. Guia para organizadores e planejadores. Brasil: Unicef, 1980.

PACHECO, A. L.; DUPRET, L. Creche: Desenvolvimento ou sobrevivência? Psicologia USP, n. 15, p. 22-30, 2004.

PÉREZ-LÓPEZ, J. Modelos explicativos del desarrollo aplicados a la atención temprana. In: PÉREZ-LÓPEZ, J.; BRITO, A. G. (Ed.). Manual de Atención Temprana. Madrid: Pirámide, 2004. 
; MARTÍNEZ-FUENTES, M. T.; DÍAZ-HERRERO, A.; BRITO DE LA NUEZ, A. Prevención, promoción del desarrollo infantil y atención temprana. Revista de Atención Temprana, n. 9, p. 77-84, 2006.

RAPOPORT, A. Da gestação ao primeiro ano de vida do bebê: apoio social e ingresso na creche. 226 f. Tese (Doutorado em Psicologia do Desenvolvimento) - Instituto de Psicologia, Universidade Federal do Rio Grande do Sul, Porto Alegre, 2003.

ROSSETTI-FERREIRA, M. C.; RAMON, F.; SILVA, A. P. Políticas de atendimento à criança pequena nos países em desenvolvimento. Cadernos de Pesquisa, n. 115, p. 32-46, 2002.

SHONKOFF, J.; MEISELS, S. (Ed.). Handbook of early intervention. Cambridge: Cambridge University Press, 2000.

SOEJIMA, C. S. Atenção e estimulação precoce relacionadas ao desenvolvimento da criança de zero a três anos de idade no ambiente da creche. $135 \mathrm{f}$. Tese (Doutorado em Educação) - Setor de Educação, Universidade Federal do Paraná, Curitiba, 2008.

SOUZA, N. Concepções de educadoras de creche sobre o desenvolvimento da criança na faixa etária de zero a três anos 97 f. Dissertação (Mestrado em Educação) - Setor de Educação, Universidade Federal do Paraná, Curitiba, 2008.

VOLPATO, C.; MELLO, S. Trabalho e formação dos educadores de creche em Botucatu: Reflexões críticas. Cadernos de Pesquisa, n. 35, p. 18-26, 2005.

VOTRUBA-DRZAL, E.; COLEY, R.; CHASE-LANSDALE, P. Child care and lowincome children's development: direct and moderated effects. Child Development, n. 75, p. 296-312, 2004.

Texto recebido em 18 de julho de 2011.

Texto aprovado em 30 de setembro de 2011. 